\title{
Computational Analysis of Potential Key Genes Associated with Dopamine Neurotransmission in Pheochromocytoma and Paraganglioma
}

\section{Orcun Avsar ${ }^{(1 D}$}

Hitit University, Department of Molecular Biology and Genetics, Corum,!Turkey

\section{ABSTRACT}

P heochromocytoma and Paraganglioma (PCPG) are rare and potentially lethal neuroendocrine tumors. PCPG that predominantly or exclusively produce and secrete DA is rarely seen, and it has been known that exclusively dopamine-secreting PCPG is related to advanced malignant features and metastases. Up to the present, little has been known about the role of dopamine neurotransmission and the dopaminergic system in the initiation and progression of PCPG. The genes with significant expression differences between normal tissue and pheochromocytoma and paraganglioma, survival and correlation analysis, CpG islands prediction, and miRNA-target enrichment analysis were performed by several bioinformatics tools. In the present study, it was determined that the COMT gene was significantly less expressed in PCPG than in normal tissue and the COMT gene showed a remarkable relationship between differential expression with shorter overall survival among the individuals with PCPG $(\mathrm{HR}=1, \mathrm{p}=0.011)$. MAOA and COMT gene pair was significantly correlated with PCPG ( $p=0.012$; $R=0.19)$, and hsa-miR-5000-5p regulates the expression of both COMT and MAOA genes ( $\mathrm{p}=0.00215, \mathrm{FDR}=0.127)$. Our findings suppose that COMT may potentially be implicated in tumor suppressive mechanism. The expression values of COMT and MAOA genes, and hsa-miR-5000-5p may have the potential to be used in the genetic evaluation of the pathogenesis and prognosis of PCPG. Further in vitro and in vivo studies are required to clarify the molecular mechanism of the dopaminergic system in the pathogenesis and prognosis of PCPG.

Keywords:

Dopamine; Pheochromocytoma and Paraganglioma (PCPG); Dopaminergic system; Gene expression; Neurotransmission

\section{INTRODUCTION}

$\mathrm{P}$ heochromocytoma and Paraganglioma (PCPG) are rare chromaffin cell tumors that produce catecholamines (CA) and present a therapeutic and diagnostic challenge, and are associated with severe morbidity when non-diagnostic $[1,2]$. Pheochromocytomas and Paragangliomas are originated from adrenal tissues or extra-adrenal parasympathetic or sympathetic paraganglia, respectively [2]. PCPG produce and secrete $\mathrm{CA}$ in the neuroendocrine system. PCPG may lead to the elevation of blood pressure and subsequently damage of target organs such as the brain, heart, and kidney. The symptoms of PCPG include palpitations, hypertension, headache, anxiety, and profuse sweating. Furthermore, PCPG may lead to metabolic disorders that are associated with insulin and blood sugar, therefore endanger the life of patients $[3,4]$.

Circulating catecholamines are composed of nore- pinephrine (NE), epinephrine (EPI), and dopamine (DA) that are mainly synthesized and released from the adrenal medulla (chromaffin cells) [5]. Specifically, DA acts as a neurotransmitter and neurohormone that modulates cognition, motivation, food intake, appetite, sexual behavior, emotion, reward system, motor activity, and prolactin secretion in the central nervous system $[6,7]$. Moreover, DA participates in the regulation of inhibition of aldosterone secretion, vasodilation, inhibition of insulin secretion, diuresis, phagocytic inhibition, IgM and IgG secretion, and natriuresis in the periphery [6].

Increased generation of catecholamines is the distinctive biochemical feature of PCPG. Until recently, the biochemical prediction of PCPG was based primarly on detecting NE, EPI, or their metabolites (no interest in DA or its metabolites). PCPG that exclusively produce and secrete DA are rarely seen, and it has been known that exclusively dopamine-secreting PCPG are related 
to advanced malignant features. Lack of clinical symptoms and the rarity of the tumor make it difficult to be detected and may cause to delay of diagnosis and poorer prognosis [8, 9]. Delayed diagnosis might be associated with the increased incidence of malignancy and metastasis. Up to the present, little has been known about the role of dopamine neurotransmission and the dopaminergic system in the initiation and progression of PCPG. Furthermore, differential diagnosis might be achieved by analyzing the expression levels of dopamine neurotransmission-related genes. Therefore, the particular relationship between dopamine neurotransmission and PCPG behavior needs further elucidating. In the present study, we aimed to determine the relationship between the genes associated with dopamine neurotransmission and PCPG by computational methods.

\section{MATERIALS AND METHODS}

\section{Expression and Survival Analysis of Target Genes in PCPG and Normal Tissue}

The sequence information and the functions of the target genes that are associated with dopamine neurotransmission were analyzed and extracted from National Center for Biotechnology Information (NCBI) database [10]. The genes with remarkable expression differences between normal tissue and pheochromocytoma and paraganglioma were designated via Gene Expression Profiling Interactive Analysis (GEPIA) database. GEPIA is an online tool and based on The Genotype-Tissue Expression (GTEx) and The Cancer Genome Atlas (TCGA) that delivers cancer and normal tissue gene expression and interactive analysis data. Furthermore, the 95\% confidence interval (95\% CI) and the Cox proportional hazard ratio (HR) of the survival plot are provided by the database [11]. Analysis of differential gene expression ensures to find the tumor-specific genes by comparing normal and tumor groups.

\section{Correlation Analysis of Some Target Genes in PCPG}

The statistical analysis of the relationship between MAOA and COMT genes and PCPG; TH and DDC genes and PCPG were verified by the use of the Spearman correlation test.

\section{Estimation of Protein-Protein Interactions}

The Search Tool for the Retrieval of Interacting Genes/ Proteins (STRING) web tool was used to predict the functional partners of the proteins that are encoded by the target genes. The significant integration and assessment of protein-protein interactions with functional (in- direct) and physical (direct) associations are provided by the STRING database [12].

\section{Prediction of CpG Islands of Target Genes}

In this study, the CpG islands in some target genes were analyzed by the MethPrimer bioinformatics tool to predict the effect of methylation on the expression of target genes. The values of "observed/expected CpG ratio"= 0.60, "island size" > 100 nucleotide, and "percentage of G plus C" $=50.0$ were set in the MethPrimer program as standard values. MethPrimer is a program that finds the potential CpG islands based on DNA sequence and designs the primers for bisulfite sequencing PCR and methylation specific PCR [13].

\section{miRNA-Target Enrichment Analysis}

microRNAs are implicated in the regulation of gene expression. Multiple genes can be regulated by one miRNA and one gene can be targeted by several miRNAs in a simultaneous way. MicroRNA ENrichment TURned NETwork (MIENTURNET) program was used for the miRNA-target enrichment analysis. miRNA-target enrichment analysis is a standard method to clarify the hierarchical effects of microRNAs in the regulatory networks of genes $[14,15]$.

\section{RESULTS AND DISCUSSION}

\section{Expression and Survival Analysis of Dopamine Neurotransmission-Related Genes in PCPG and Normal Tissue by in silico Methods}

The expression values of TH, DDC, DBH, PNMT, MAOA, MAOB, COMT, ALDH2, DRD1, DRD2, DRD3, DRD4, DRD5, SLC6A3, SLC18A1 and SLC18A2 genes were analyzed and expression differences between PCPG and normal tissue were compared. The genes with remarkable expression differences between PCPG and normal tissue were identified. According to the analysis, MAOA, MAOB, COMT, ALDH2, and DRD1 genes were significantly less expressed in PCPG than in normal tissue. On the other hand, expression of TH, DDC, DBH, PNMT, DRD2, SLC18A1, and SLC18A2 genes were significantly higher in PCPG compared to normal tissue (Table 1). The median, $\log 2$, and percentage values of the over-expressed genes in PCPG were shown in Table 2. The comparison of the expression values of the genes in PCPG and normal tissue was illustrated in Figure 1, Figure 2, and Figure 3. The relationship between gene expressions with overall survival of PCPG patients were evaluated to determine the role of the genes associated with the dopaminergic system in PCPG prognosis by dividing the 
population into low expression and high expression, only one gene showed a remarkable relationship between differential expression with shorter overall survival among the individuals with PCPG. Log rank $(\mathrm{p}<0.05)$ is accepted as statistically significant. The gene-COMT $(\mathrm{HR}=1$, $\mathrm{p}=0.011)$ may serve as a molecular biomarker for PCPG (Figure 4).

\section{Correlation Analysis of MAOA and COMT Genes and TH and DDC Genes in PCPG}

The statistical analysis of the relationship between MAOA and COMT genes (encode the enzymes that degrade dopamine) and PCPG was performed via the GEPIA database. It was determined that MAOA and COMT gene pair was significantly correlated with PCPG by the Spearman correlation analysis $(\mathrm{p}=0.012 ; \mathrm{R}=0.19)$ (Figure 5). Furthermore, Spearman correlation analysis has determined that the TH and DDC gene (encode the enzymes that are involved in the synthesis of dopamine) pair was significantly associated with PCPG $(p=0.043$; $\mathrm{R}=0.15$ ) (Figure 6).

\section{Prediction of Protein-Protein Interactions}

Protein-protein interactions were estimated by STRING web tool to elucidate the functional partners of the enzymes degrading DA (MAOA and COMT) and the enzymes catalyzing the synthesis of DA (TH and DDC). Based on the findings of STRING for MAOA protein, functional interactants with high confidence were determined as: COMT (0.989), DDC (0.971), DBH (0.962), ALDH2 (0.961), CYP2D6 (0.955), AOC2 (0.951), ALDH3B2 (0.945), HNMT (0.943), ADH1B (0.942), PNMT (0.940) (Figure 7). Functional interactants of COMT protein were predicted as follows: MAOA (0.989), MAOB (0.987), DDC (0.980), DBH (0.971), CYP1B1 (0.971), CYP1A1 (0.968), PNMT (0.963), ALDH2 (0.944), ALDH1B (0.944), DRD2 (0.940) (Figure 8). Functional partners of TH protein were determined as follows: SNCA (0.992), DDC (0.992), SPR (0.968), PCBD1 (0.953), QDPR (0.951), DBH (0.945), TYR (0.943), SLC18A2 (0.937), AKR1B1 (0.927), GOT1 (0.926) (Figure 9). The functional interactants of DDC protein with high confidence were determined as: TH (0.992), DBH (0.987), TPH1 (0.984), COMT (0.980), MAOB (0.975), MAOA (0.971), TPH2 (0.969), PAH (0.966), AOC1 (0.964), AANAT (0.962) (Figure 10). According to the predictions of STRING web tool, it was demonstrated that MAOA and $\mathrm{TH}$ interact with COMT protein, and DDC, respectively.

\section{Analysis of CpG Islands}

Methylation is involved in the regulation of gene expression [16]. In the present study, CpG islands were analy- zed to determine the potential role of methylation in the regulation of $\mathrm{TH}, \mathrm{DDC}, \mathrm{MAOA}$, and COMT genes. According to the findings of the study, the number of $\mathrm{CpG}$ islands (CIG) were indicated as follows: TH-4 CIG; DDC1 CIG; MAOA-0 CIG; COMT-4 CIG, and the results are seen in Table 3.

\section{miRNA-Target Enrichment Analysis}

The miRNAs which have the potential to regulate the expression of more than one gene associated with dopaminergic system among the investigated mRNAs were shown in Table 4. Common miRNAs for SLC18A2 and DRD2: hsa-miR-141-3p and hsa-miR-200a-3p; for SLC18A2 and DRD1: hsa-miR-142-5p and hsa-miR-55903p; for DRD2 and SLC18A2: hsa-miR-9-5p; for COMT, SLC6A3, and DRD1: hsa-miR-30a-5p, hsa-miR-30b-5p, hsa-miR-30c-5p, hsa-miR-30d-5p, and hsa-miR-30e-5p; for COMT and MAOA: hsa-miR-5000-5p; for ALDH2 and TH: hsa-miR-1-3p; for ALDH2, DBH, DRD5, and MAOB: hsa-miR-335-5p; for ALDH2 and COMT: hsa-

Table 1. Expression values of the genes associated with dopamine neurotransmission in PCPG and normal tissue

\begin{tabular}{|c|c|c|}
\hline Gene ID & $\begin{array}{c}\text { Pheochromocytoma and Para- } \\
\text { ganglioma (PCPG) }\end{array}$ & Normal tissue \\
\hline $\mathrm{TH}^{*}$ & $1,830.03$ & 24.87 \\
\hline $\mathrm{DDC}^{*}$ & 599.55 & 3.34 \\
\hline $\mathrm{DBH}^{\circ}$ & $2,853.17$ & 20.21 \\
\hline $\mathrm{PNMT}^{*}$ & 113 & 8.91 \\
\hline $\mathrm{MAOA}^{*}$ & 6.97 & 22.01 \\
\hline $\mathrm{MAOB}^{*}$ & 8.26 & 39.2 \\
\hline $\mathrm{COMT}^{*}$ & 112.21 & 256.67 \\
\hline ALDH2* & 69 & 346.45 \\
\hline DRD1* & 0.05 & 0.24 \\
\hline DRD2* & 86.66 & 0.98 \\
\hline DRD3 & - & - \\
\hline DRD4 & 0.32 & 0.19 \\
\hline DRD5 & 0.02 & 0.01 \\
\hline SLC6A3 & 0.02 & 0.01 \\
\hline SLC18A1* & 145.98 & 0.37 \\
\hline SLC18A2 & 133.17 & 1.03 \\
\hline
\end{tabular}

miR-16-5p; for PNMT and DRD3: hsa-miR-26b-5p.

Pheochromocytoma and Paraganglioma (PCPG) are rare and potentially lethal neuroendocrine tumors. Despite most of the tumors are benign, approximately $10-15 \%$ are classified as malignant and develop metastases in nonchromaffin tissues such as bone, liver, and lymph nodes [17]. It is 
Table 2. The median, $\log 2$, and percentage values of the over-expressed genes in PCPG

\begin{tabular}{|c|c|c|c|c|}
\hline Gene ID & Median (Tumor) & Median (Normal) & Log2 (Fold change) & Percentage \\
\hline $\mathrm{TH}$ & 4539.601 & 616.945 & 2.877 & $1.00 \mathrm{e}+0$ \\
\hline DDC & 1548.065 & 385.690 & 2.002 & $1.00 \mathrm{e}+0$ \\
\hline $\mathrm{DBH}$ & 6086.722 & 303.910 & 4.319 & $1.00 \mathrm{e}+0$ \\
\hline PNMT & 2178.772 & 465.310 & 2.225 & $9.44 \mathrm{e}-1$ \\
\hline SLC18A1 & 759.997 & 20.487 & 5.146 & $1.00 \mathrm{e}+0$ \\
\hline SLC18A2 & 534.371 & 89.529 & 2.564 & $1.00 \mathrm{e}+0$ \\
\hline
\end{tabular}
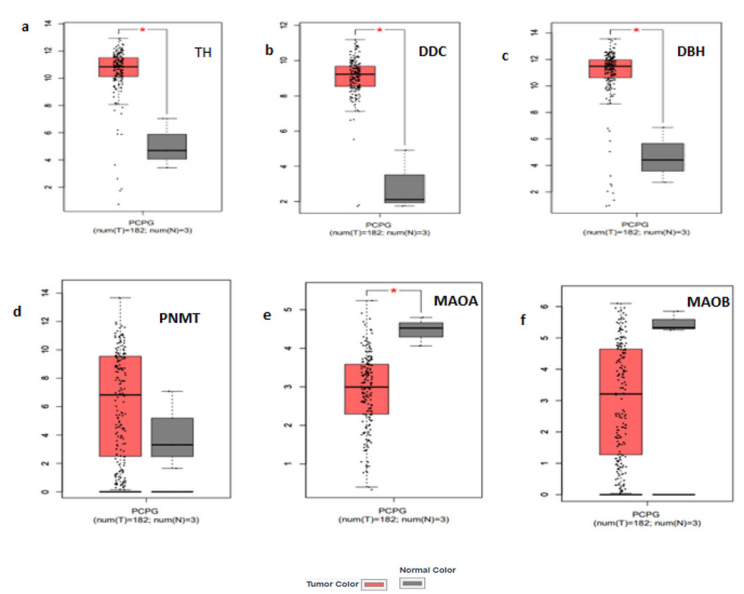

Figure 1. Differential expressions of TH, DDC, DBH, PNMT, MAOA, and MAOB genes in PCPG
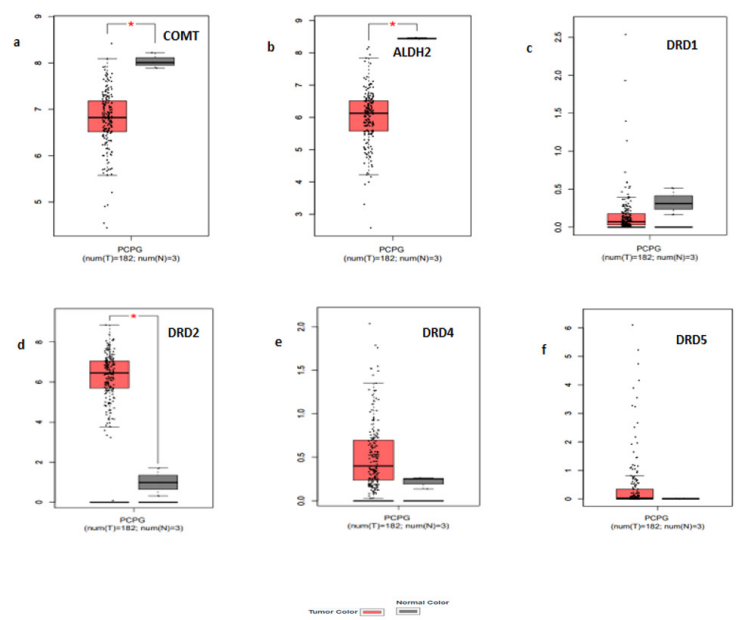

Figure 2. Differential expressions of COMT, ALDH2, DRD1, DRD2, DRD4, and DRD5 genes in PCPG

significant to diagnose PCPG in an accurate and early way to treat patients and also affected members in family cases [18]. PCPG usually secrete high amounts of catecholamines. Tumors that predominantly or exclusively produce DA are rare and often malignant and metastatic $[17,19]$.

Dopamine, biogenic monoamine, is a member of catecholamine neurotransmitters and generated in both central nervous system and periphery. The dopaminergic system is implicated in several biological functions such
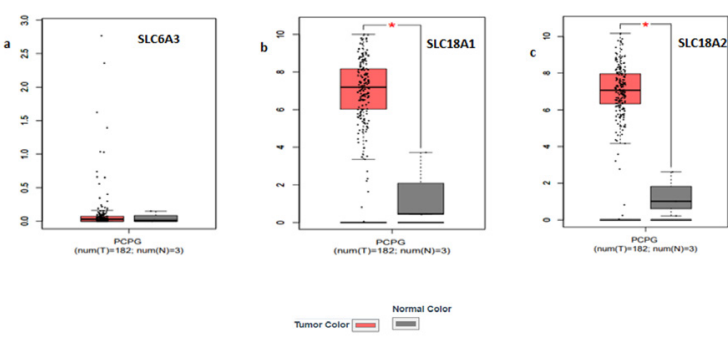

Figure 3. Differential expressions of SLC6A3, SLC18A1, and SLC18A2 genes in PCPG

as motivation, cognition, motor activity, maternal behavior, reward system, and reproductive behavior. Dopamine is synthesized directly from tyrosine or indirectly from L-phenylalanine. Tyrosine hydroxylase is the rate limiting enzyme in DA synthesis that converts tyrosine to L-DOPA and L-DOPA may be converted into DA by DOPA decarboxylase. Subsequently, DA is sequestered into the synaptic vesicles through vesicular monoamine transporter 2 (VMAT2) in dopaminergic neurons, preventing oxidation. DA may be further converted into NE or EPI by modifications from DBH and PNMT in adrenergic and noradrenergic cells [20]. In a non-acidic microenvironment, DA is metabolized by MAO and COMT enzymes [21]. Dopamine exerts its functions via binding to $G$ protein-coupled receptors (DRD1, DRD2, DRD3, DRD4, DRD5) [22].

The dopaminergic phenotype of PCPG is composed of a rare subtype of PCPG that predominantly or exclusively secrete DA (no significant NE and EPI levels) [23]. In contrast to the symptoms that are seen in epinephrine and norepinephrine-secreting tumor in high amounts, the tumors that predominantly or exclusively produce DA are normotensive and asymptomatic [23]. DA secreting PCPG are generally larger tumors than epinephrine and norepinephrine-secreting tumors due to delayed diagnosis (incidentally identified). There are several significant differences, such as the risk of recurrence, malignancy, and peri-operative cardiovascular collapse between DA secreting PCPG and NE and EPI secreting PCPG in terms of management [24]. Moreover, DA secreting PCPG have high malignancy risk compared to NE and EPI secreting PCPG [25].

In the current study, we have analyzed the genes as- 

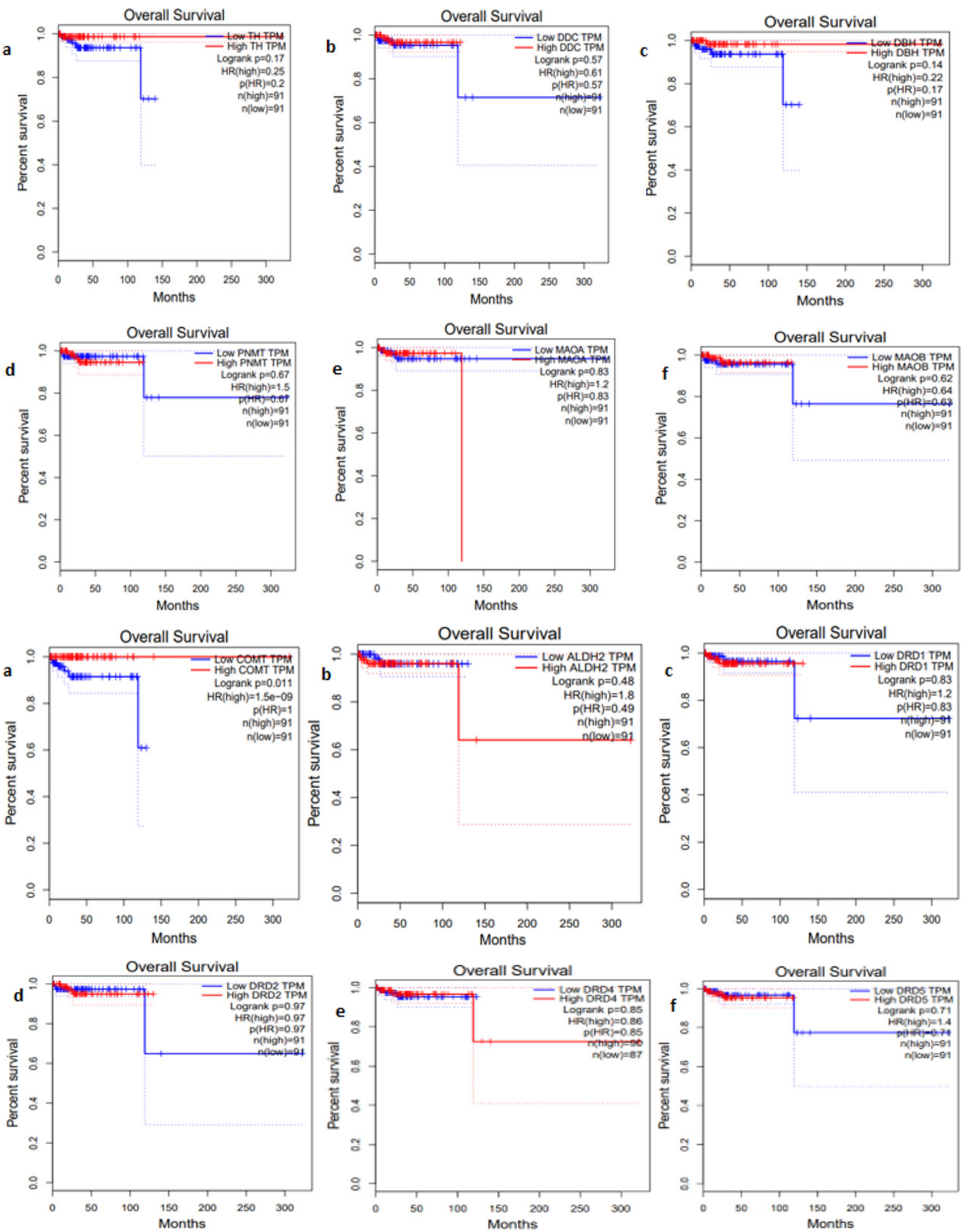

Figure 4. Associations of the genes that are associated with dopamine neurotransmission with overall survival in PCPG

sociated with dopamine neurotransmission and specifically focused on the genes that encode the main enzymes involved in DA synthesis and metabolism. High DA levels have been demonstrated in metastatic paragangliomas in consequence of reduced expression level of DBH [26]. In a study conducted with 21 PCPG patients, lower MAOA and
COMT expression levels were detected in tumor tissues [27]. It has been reported that the amounts of mRNA encoded by $\mathrm{TH}, \mathrm{DDC}$, and DBH genes were higher in pheochromocytomas compared to the normal adrenal medulla, whereas the PNMT mRNA levels were higher in the normal tissue [28]. According to the survival analysis of the current study, 

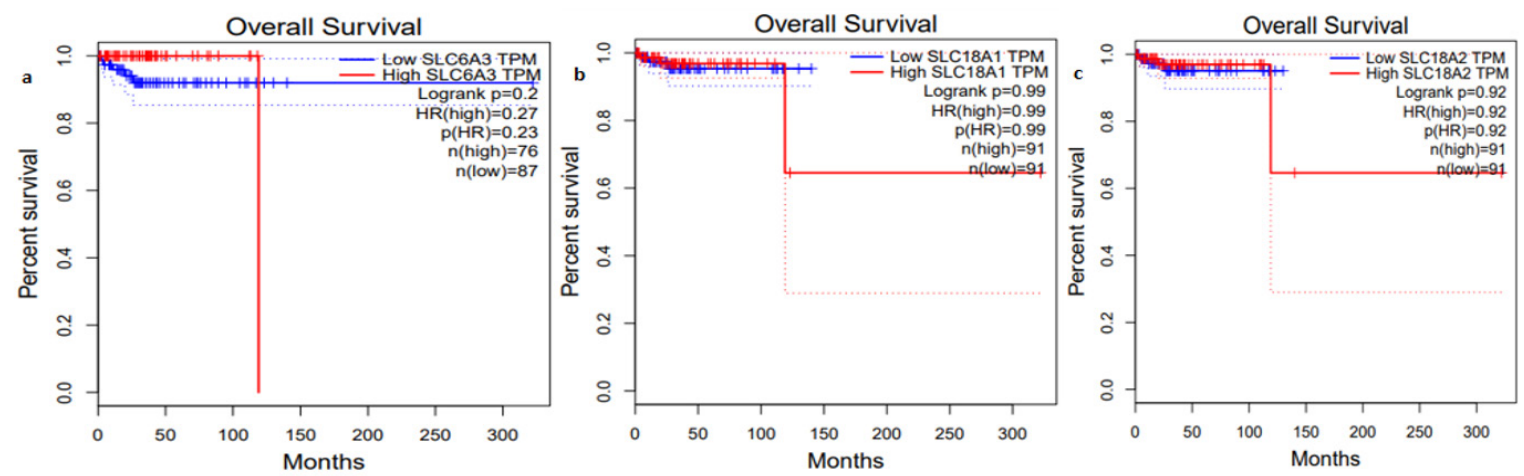

Figure 4. Continued

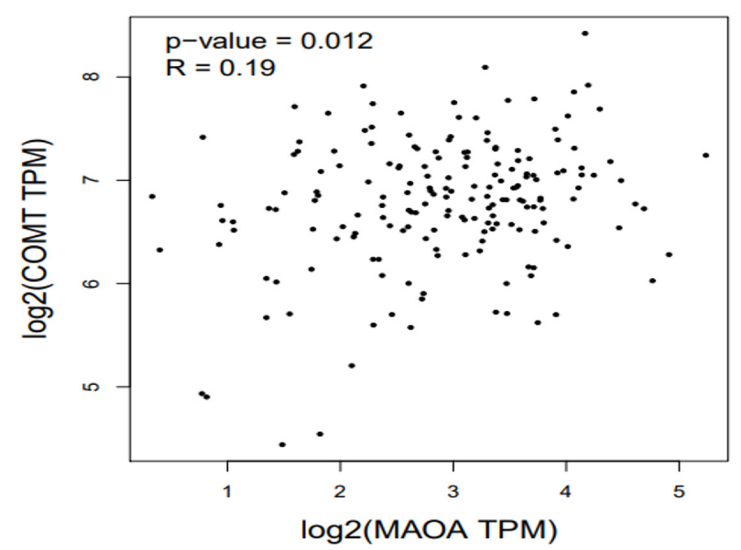

Figure 5. Spearman correlation analysis of MAOA and COMT genes with PCPG

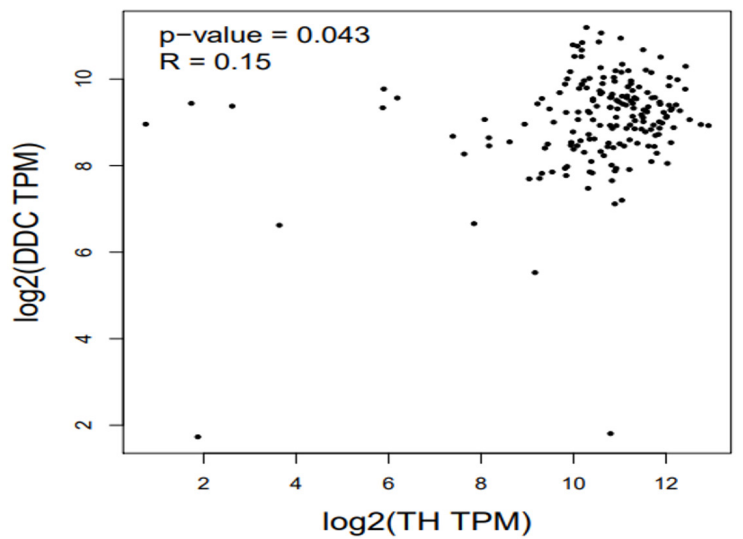

Figure 6. Spearman correlation analysis of TH and DDC genes with PCPG

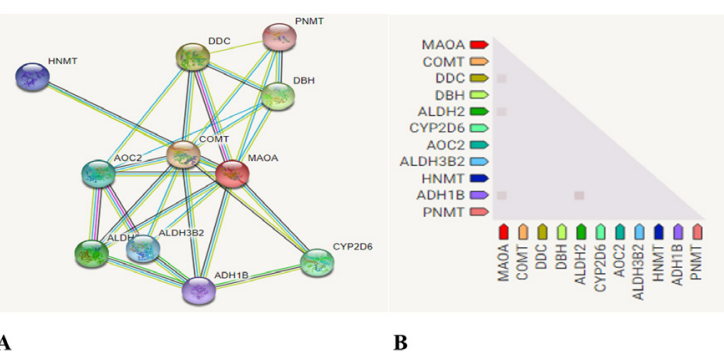

Figure 7. A. The predicted partners of MAOA B. Coexpression pattern of the genes that correlates with the gene encodes MAOA

the COMT gene was significantly less expressed in PCPG
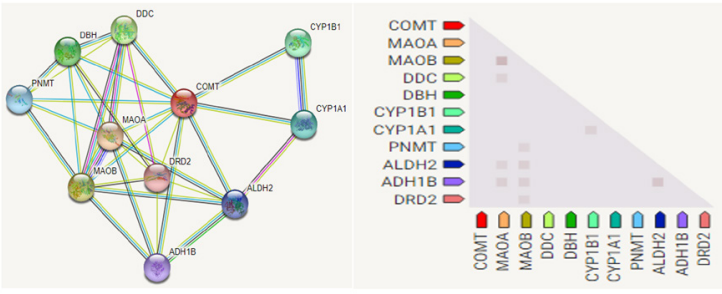

A

B

Figure 8. A. The predicted partners of COMT B. Coexpression pattern of the genes that correlates with the gene encodes COMT

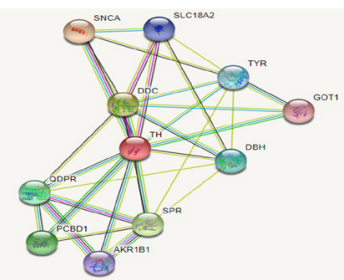

A

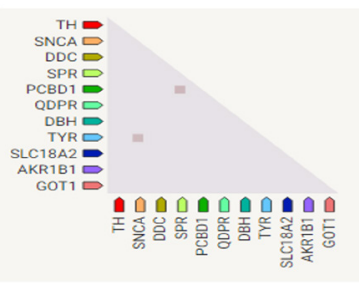

B

Figure 9. A. The predicted partners of TH B. Coexpression pattern of the genes that correlates with the gene encodes $\mathrm{TH}$
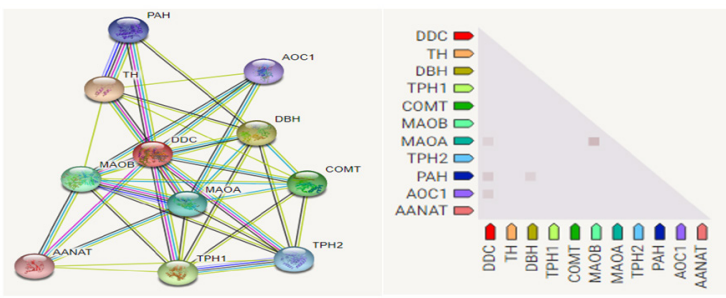

A B

Figure 10. A. The predicted partners of DDC B. Coexpression pattern of the genes that correlates with the gene encodes DDC

compared to the normal tissue. Therefore, COMT may serve as a molecular biomarker for PCPG. DNA methylation and miRNAs directly can modulate gene expression. Based on the findings of the present study, the COMT gene has 4. CpG islands that are associated with DNA methylation. COMT protein significantly interacts with MAOA protein. hsa-miR-5000-5p regulates the expression of both COMT and MAOA genes $(\mathrm{p}=0.00215, \mathrm{FDR}=0.127)$. In this regard, our findings suppose that COMT may potentially be implicated in tumor suppressive mechanism in PCPG. In patients with dopamine secreting PCPG, post-surgical observation 
Table 3. The position and features of CpG islands of TH, DDC, MAOA, COMT genes

\begin{tabular}{|c|c|c|c|c|c|c|}
\hline Gene & Island no & Island size & Island start & Island end & GC\% percent & $\mathrm{O} / \mathrm{E}$ ratio \\
\hline \multirow{4}{*}{$\mathrm{TH}$} & Island 1 & 120 & 103 & 222 & 50.0 & 0.6 \\
\hline & Island 2 & 198 & 292 & 489 & 50.0 & 0.6 \\
\hline & Island 3 & 604 & 583 & 1186 & 50.0 & 0.6 \\
\hline & Island 4 & 193 & 1273 & 1465 & 50.0 & 0.6 \\
\hline DDC & Island 1 & 257 & 132 & 388 & 50.0 & 0.6 \\
\hline \multirow[t]{2}{*}{ MAOA } & None & & & & & \\
\hline & Island 1 & 142 & 16 & 157 & 50.0 & 0.6 \\
\hline \multirow{3}{*}{ COMT } & Island 2 & 180 & 367 & 546 & 50.0 & 0.6 \\
\hline & Island 3 & 99 & 605 & 703 & 50.0 & 0.6 \\
\hline & Island 4 & 189 & 773 & 961 & 50.0 & 0.6 \\
\hline
\end{tabular}

Table 4. miRNA-target enrichment analysis result for the genes associated with dopamine neurotransmission

\begin{tabular}{|c|c|c|c|c|c|c|c|c|}
\hline miRNA family & p-value & FDR & Odd ratio & $\begin{array}{l}\text { Number } \\
\text { of inte- } \\
\text { ractions }\end{array}$ & $\begin{array}{l}\text { T a r g e t } \\
\text { Gene } 1\end{array}$ & $\begin{array}{l}\text { T a r g e t } \\
\text { Gene } 2\end{array}$ & $\begin{array}{l}\text { Target } \\
\text { Gene } 3\end{array}$ & $\begin{array}{c}\text { Target } \\
\text { Gene } 4\end{array}$ \\
\hline hsa-miR-141-3p/hsa-miR-200a-3p & 0.175 & 0.774 & 0.382 & 2 & SLC18A2 & DRD2 & & \\
\hline hsa-miR-142-5p/hsa-miR-5590-3p & 0.189 & 0.774 & 0.401 & 2 & SLC18A2 & DRD1 & & \\
\hline hsa-miR-9-5p & 0.330 & 0.774 & 0.586 & 2 & DRD2 & SLC18A2 & & \\
\hline $\begin{array}{l}\text { hsa-miR-30a-5p/hsa-miR-30b-5p/hsa-miR-30c-5p/ } \\
\text { hsa-miR-30d-5p/hsa-miR-30e-5p }\end{array}$ & 0.139 & 0.774 & 0.443 & 3 & COMT & SLC6A3 & DRD1 & \\
\hline hsa-miR-5000-5p & 0.00215 & 0.127 & 0.0349 & 2 & COMT & MAOA & & \\
\hline hsa-miR-1-3p & 0.233 & 0.280 & 0.459 & 2 & ALDH2 & $\mathrm{TH}$ & & \\
\hline hsa-miR-335-5p & 0.258 & 0.293 & 0.654 & 4 & ALDH2 & $\mathrm{DBH}$ & DRD5 & MAOB \\
\hline hsa-miR-16-5p & 0.469 & 0.494 & 0.775 & 2 & ALDH2 & COMT & & \\
\hline hsa-miR-26b-5p & 0.573 & 0.593 & 0.933 & 2 & PNMT & DRD3 & & \\
\hline
\end{tabular}

and genetic assessment has a notable effect on the prognosis of the disease. The expression values of COMT and MAOA genes, related CpG islands, and hsa-miR-5000-5p may have the potential to be used in the genetic evaluation of the pathogenesis and prognosis of PCPG. Further research is required to further enlighten the relationship between dopamine neurotransmission and the carcinogenesis of PCPG.

\section{CONCLUSION}

The present study reported that the COMT gene is significantly associated with PCPG. The findings of in silico analysis demonstrated that COMT might potentially be implicated in tumor suppressive mechanism. Delayed diagnosis and poorer prognosis are still a problem, and some of the tumors are recognized at autopsy. Therefore, expression analysis may be considered as a component of the clinical evaluation of patients. It requires further in vitro and in vivo dopamine neurotransmission studies in order to understand the molecular mechanism of the dopaminergic system in the pathogenesis of PCPG and its metastases features.

\section{CONFLICT OF INTEREST}

Authors approve that to the best of their knowledge, there is not any conflict of interest or common interest with an institution/organization or a person that may affect the review process of the paper.

\section{References}

1. Jochmanova I, Pacak K. Genomic landscape of pheochromocytoma and paraganglioma. Trends Cancer 4 (2018) 6-9.

2. Kantorovich V, Pacak K. New insights on the pathogenesis of paraganglioma and pheochromocytoma [version 1; referees: 2 approved]. F1000Research 7 (2018) F1000.

3. Su Q, Ding Q, Zhang Z, Yang Z, Qiu Y, Li X, Mo W. Identification of genes associated with the metastasis of pheochromocytoma/ paraganglioma based on weighted gene coexpression network analysis. BioMed Research International 2020 (2020) 3876834.

4. Neumann HPH, Young WF Jr, Eng C. Pheochromocytoma and 
paraganglioma. The New England Journal of Medicine 381 (2019) 552-565.

5. Mahata SK, Zheng H, Mahata S, Liu X, Patel KP. Effect of heart failure on catecholamine granule morphology and storage in chromaffin cells. Journal of Endocrinology 230 (2016) 309-323.

6. Osinga TE, Links TP, Dullaart RPF, Pacak K, van der HorstSchrivers ANA, Kerstens MN, Kema IP. Emerging role of dopamine in neovascularization of pheochromocytoma and paraganglioma FASEB Journal 31 (2017) 2226-2240.

7. Avsar O, Kuskucu A, Sancak S, Genc E. Are dopaminergic genotypes risk factors for eating behavior and obesity in adults? Neuroscience Letters 654 ( 2017) 28-32.

8. Yi JW, Oh EM, Lee KE, Choi JY, Koo do H, Kim KJ, Jung KC, Kim SY, Youn YK. An exclusively dopamine secreting paraganglioma in the retroperitoneum: a first clinical case in Korea. Journal of the Korean Surgical Society 82 (2012) 389-393.

9. Foo SH, Chan SP, Ananda V, Rajasingam V, Rajasingam V. Dopamine-secreting phaeochromocytomas and paragangliomas: clinical features and management. Singapore Medical Journal 51 (2010) e89.

10. Sayers EW, Agarwala R, Bolton EE, Brister JR, Canese K, Clark K, Connor R, Fiorini N, Funk K, Hefferon T, Holmes JB, Kim S Kimchi A, Kitts PA, Lathrop S, Lu Z, Madden TL, Marchler-Bauer A, Phan L, Schneider VA, Schoch CL, Pruitt KD, Ostell J. Database resources of the National Center for Biotechnology Information. Nucleic Acids Research 47 (2019) D23-D28

11. Tang Z, Li C, Kang B, Gao G, Li C, Zhang Z. GEPIA: a web server for cancer and normal gene expression profiling and interactive analysis. Nucleic Acid Research 45 (2017) W98-W102.

12. Szklarczyk D, Franceschini A, Wyder S, Forslund K, Heller D, Huerta-Cepas J, Simonovic M, Roth A, Santos A, Tsafou KP, Kuhn M, Bork P, Jensen LJ, von Mering J. STRING v10: protein-protein interaction networks, integrated over the tree of life. Nucleic Acids Research 43 (2015) D447-D452.

13. Li LC, Dahiya R. MethPrimer: designing primers for methylation PCRs. Bioinformatics 18 (2002) 1427-1431.

14. Licursi V, Conte F, Fiscon G, Paci P. MIENTURNET: an interactive web tool for microRNA-target enrichment and network-based analysis. BMC Bioinformatics 20 (2019) 545.

15. Xu J, Wong CW. Enrichment Analysis of miRNA Targets, in: Ying SY. (Eds.). MicroRNA Protocols. Methods in Molecular Biology (Methods and Protocols), Humana Press, Totowa, NJ, pp. 91-103, 2013.
16. Moore L, Le T, Fan G. DNA methylation and its basic function. Neuropsychopharmacology 38 (2013) 23-38.

17. van Berkel A, Lenders JW, Timmers HJ. Biochemical diagnosis of phaeochromocytoma and paraganglioma. European Journal of Endocrinology 170 (2014) R109-R119.

18. Tutal E, Arslan MS. Diagnosis of pheochromocytoma and paraganglioma. Endocrinology and Diabetes Open Access 1 (2018) 105.

19. Soh AW, Kek PC. Dopamine-secreting carotid body paragangliomas-biochemical control with radiotherapy. Internal Medicine 51 (2012) 613-618.

20. Klein MO, Battagello DS, Cardoso AR, Hauser DN, Bittencourt JC, Correa RG. Dopamine: Functions, signaling, and association with neurological diseases. Cellular and Molecular Neurobiology 39 (2019) 31-59.

21. Finberg JPM. Inhibitors of MAO-B and COMT: their effects on brain dopamine levels and uses in Parkinson's disease. Journal of Neural Transmission 126 (2019) 433-448.

22. Beaulieu JM, Gainetdinov RR. The physiology, signaling, and pharmacology of dopamine receptors. Pharmacological Reviews 63 (2011) 182-217.

23. Gupta G, Pacak K, AACE Adrenal Scientific Committee. Precision medicine: an update on genotype/biochemical phenotype relationships in pheochromocytoma/paraganglioma patients. Endocrine Practice 23 (2017) 690-704.

24. Bozin M, Lamb A, Putra LJ. Pheochromocytoma with negative metanephrines: A rarity and the significance of dopamine secreting tumors. Urology Case Reports 12 (2017) 51-53.

25. Foo SH, Chan SP, Ananda V, Rajasingam V. Dopamine-secreting pheochromocytoma and paragangliomas: clinical features and management. Singapore Medical Journal 51 (2010) 89.

26. Konosu-Fukaya S, Omata K, Tezuka Y, Ono Y, Aoyama Y, Satoh F, Fujishima F, Sasano H, Nakamura Y. Catecholamine synthesizing enzymes in pheochromocytoma and extraadrenal paraganglioma. Endocrine Pathology 29 (2018) 302-309.

27. Grouzmann E, Matter M, Bilz S, Herren A, Triponez F, Henzen C, Kim KS, Zulewski H, Buclin T, Brakch N, Abid K. Monoamine oxidase A down-regulation contributes to high metanephrine concentration in pheochromocytoma. Journal of Clinical Endocrinology and Metabolism 97 (2012) 2773-2781.

28. Isobe K, Nakai T, Yukimasa N, Nanmoku T, Takekoshi K, Nomura F. Expression of mRNA coding for four catecholamine synthesizing enzymes in human adrenal pheochromocytomas. European Journal of Endocrinology 138 (1998) 383-387. 